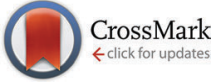

Cite this: DOI: $10.1039 / c 6 c p 04438 g$

\title{
Direct oxidative pathway from amplex red to resorufin revealed by in situ confocal imaging
}

\author{
Pauline Lefrançois, ${ }^{a b}$ Venkata Suresh Reddy Vajrala, ${ }^{\text {ab }}$ Imelda Bonifas Arredondo, $\dagger^{\mathrm{c}}$ \\ Bertrand Goudeau, ${ }^{\text {ab }}$ Thomas Doneux, ${ }^{d}$ Laurent Bouffier ${ }^{\star a b}$ and \\ Stéphane Arbault*ab
}

\begin{abstract}
Amplex Red (AR) is a very useful chemical probe that is employed in biochemical assays. In these assays, the non-fluorescent AR is converted to resorufin (RS), which strongly absorbs in the visible region $\left(\lambda_{\text {abs }}=\right.$ $572 \mathrm{~nm}$ ) and yields strong fluorescence $\left(\lambda_{\text {fluo }}=583 \mathrm{~nm}\right.$ ). Even if AR is commonly used to report on enzymatic oxidase activities, an increasing number of possible interferences have been reported, thus lowering the accuracy of the so-called AR assay. As a redox-based reaction, we propose here to directly promote the conversion of AR to RS by means of electrochemistry. The process was first assessed by classic electrochemical and spectroelectrochemical investigations. In addition, we imaged the electrochemical conversion of AR to RS at the electrode surface by in situ confocal microscopy. The coupling of methodologies allowed to demonstrate that RS is directly formed from AR by an oxidation step, unlike what was previously reported. This gives a new insight in the deciphering of AR assays' mechanism and about their observed discrepancy.
\end{abstract}

Received 24th June 2016, Accepted 22nd July 2016

DOI: $10.1039 / c 6 c p 04438 g$

www.rsc.org/pccp a.

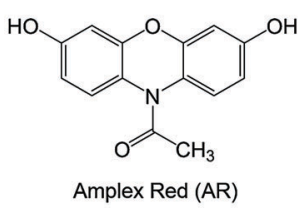

b.

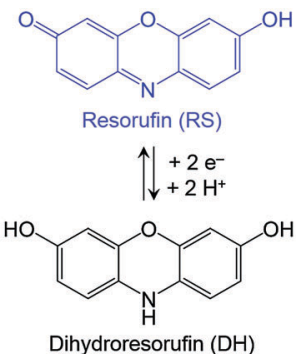

Dihydroresorufin $(\mathrm{DH})$ resorufin (RS) bearing an acetyl protecting group located on the nitrogen atom of the 1,4-oxazine ring (see Scheme 1b). An aqueous solution of RS has a deep pink colour because this chromophore does strongly absorb light in the visible region with a maximum absorption band centred at $572 \mathrm{~nm}$ and a corresponding molar absorption coefficient $\varepsilon_{\mathrm{RS}}=56000 \mathrm{M}^{-1} \mathrm{~cm}^{-1}$ at $\mathrm{pH}$ above $7.5 .^{1}$ It is also a highly fluorescent dye because upon excitation it reemits efficiently at a maximum of $583 \mathrm{~nm}$ with a typical quantum yield $\Phi_{\mathrm{RS}}=0.41$ in water at $\mathrm{pH} 8$. . $^{2}$ The molecular structure of RS comprises an electroactive iminoquinone moiety. The corresponding electrochemical behaviour is characterized by a bi-electronic reversible reduction of RS to the corresponding colourless dihydroresorufin (DH). ${ }^{3}$ Such a fluorogenic behaviour

\footnotetext{
${ }^{a}$ Univ. Bordeaux, ISM, UMR 5255, F-33400 Talence, France.

E-mail: laurent.bouffier@enscbp.fr, stephane.arbault@enscbp.fr

${ }^{b}$ CNRS, ISM, UMR 5255, F-33400 Talence, France

${ }^{c}$ Instituto Potosino de Investigación Científica y Tecnológica,

78216 San Luis Potosí, Mexico

${ }^{d}$ Chimie Analytique et Chimie des Interfaces, Faculté des Sciences, Université Libre de Bruxelles (ULB), Boulevard du Triomphe, 2, CP 255, B-1050 Bruxelles, Belgium

$\dagger$ In memory of our friend Imelda.
}

Scheme 1 (a) Chemical structure of Amplex Red (AR) and (b) electrochemical reduction of resorufin (RS) to dihydroresorufin (DH).

makes RS a very attractive redox-sensitive probe whose fluorescence drops down upon chemical reduction by a reactant or following an electron transfer at an electrode surface.

Therefore, the reduction of RS may generate a so-called fluorescence OFF-signal when used in the transduction step of a biosensor. The opposite strategy consists in using DH oxidation to RS to turn on the fluorescence signal. Unfortunately, the latter can only be performed in deaerated conditions because DH is not stable in the presence of oxygen and oxidizes spontaneously to form RS. The design and synthesis of AR was therefore proposed as a very useful alternative because it is a stable protected form of $\mathrm{DH}$ that can produce the desired ON-signal during the course of a bioassay. ${ }^{4-6} \mathrm{AR}$ is since then used as a universal redox-sensing indicator capable to report enzymatic processes involving oxidases, ${ }^{7-9}$ or measure the concentration of reactive-oxygen and -nitrogen species (ROS and RNS, respectively). ${ }^{10-12}$ In that context, 
AR was employed for the enzymatic determination of lipid molecules or fatty acids such as cholesterol, ${ }^{13}$ or measure free phosphate level in solution. ${ }^{14}$ It was equally used to study the production of ROS by mitochondria and more particularly elucidate the central role of respiratory complex III in the process. $^{15-17}$

However, despite the very popular use of AR, a certain number of precautions have to be considered to ensure precision and reliability of assays because AR stability may be problematic. First, the molecule was reported to be sensitive to photooxidation. ${ }^{18-20}$ Moreover, the presence of numerous interfering chemicals could potentially lower the efficiency of AR redox mediation. For instance, the direct interference of NADH and reduced glutathione auto-oxidation were reported. ${ }^{21}$ Also, the presence of antioxidants such as anthracyclines, ${ }^{22}$ gallic or ascorbic acids, is known to interfere as well. ${ }^{23}$ At last but not least, various oxide nanoparticles such as $\mathrm{TiO}_{2}$ or $\mathrm{Fe}_{2} \mathrm{O}_{3}$ were found to markedly skew the accuracy of $\mathrm{H}_{2} \mathrm{O}_{2}$ detection using the AR-based assay. ${ }^{24}$ Very recently, the generation of RS from AR was also used for an indirect mapping of the photocurrent generated on single-crystalline rutile titanium oxide nanorods. ${ }^{25}$

Somehow, the drawbacks of the widely-used AR assay are related to the chemical complexity of the analysis medium which is typically a buffered mixture, but this can even be more critical in the context of in vitro measurements on cells. Surprisingly, very few modern electrochemical studies have been conducted either with AR, RS or also their resazurin analogue (RZ) to decipher on their redox properties. ${ }^{26,27}$ Electrochemistry is indeed a very appropriate method to understand the thermodynamic stability of AR because the Fermi level of the electrode can be finely adjusted in order to tune the redox state of any sort of molecule. A detailed electrochemical investigation of AR conversion to RS has never been reported so far. From a chemical point of view, this conversion is an oxidation process. Practically, AR is very often used as co-reactant in enzymatic assays but the whole mechanism is rarely discussed, or marginally. In majority, AR is used in combination with oxidases or peroxidases such as the Horseradish Peroxidase (HRP). ${ }^{28}$ The mechanism appears to be rather complex and several pathways have been proposed involving the possible conversion of $\mathrm{H}_{2} \mathrm{O}_{2}$ into peroxy radicals $\mathrm{RO}^{\bullet}$ by $\mathrm{HRP}$, these radicals being capable to react with AR via an irreversible oxidation to RS. More recently, another study based on single molecule measurements at optic fibre arrays revealed that HRP may first oxidize AR to form a radical intermediate. Two radicals may then undergo an enzyme-independent disproportionation to form stoichiometric amounts of RS and AR, respectively. ${ }^{29}$ In all cases, redox enzymes are ensuring the electron transfer steps, but it is conceptually simpler to use an electrode instead for a direct redox control of the conversion. Electrochemical techniques are thus very complementary to spectroscopic investigations which are the other methods of choice for studying such fluorogenic dyes. The combination of both approaches could also give a greater level of characterization. Consequently, the present work is devoted to recording in situ spectroelectrochemistry data and acquiring fluorescence imaging at the electrode surface to study the electrochemical conversion of AR in buffered aqueous solutions.

\section{Experimental section}

\section{Chemicals}

Amplex ${ }^{\circledR}$ Red, AR ( $N$-acetyl-3,7-dihydroxyphenoxazine; ThermoFisher, FR) was solubilized in DMSO (Sigma-Aldrich, $\geq 99.5 \%$ ) to prepare a $10^{-2} \mathrm{~mol} \mathrm{~L}^{-1}$ stock solution, which was subsequently diluted at various concentrations in phosphate buffered saline (PBS: $10^{-2} \mathrm{~mol} \mathrm{~L}^{-1}$ phosphates, NaCl $0.138 \mathrm{~mol} \mathrm{~L}^{-1}$; KCl $2.7 \times$ $10^{-3} \mathrm{~mol} \mathrm{~L}^{-1}$, pH 7.4). PBS (reference P5368 from Sigma, FR) was prepared by dissolving the aforementioned salts in pure water (Milli-Q, Millipore, Integral 3, $0.22 \mu \mathrm{m}$ MilliPak filter). A fresh AR stock solution was always prepared prior to use. Resorufin, RS (7-hydroxy-3H-phenoxazin-3-one; ThermoFisher, FR) was solubilized in DMSO as a $2.5 \times 10^{-3}$ mol L ${ }^{-1}$ stock solution, subsequently diluted in PBS at the desired concentration before use.

\section{Electrochemistry}

Cyclic voltammetry and chronoamperometry measurements ( $\mu$ AutoLab III potentiostat, Metrohm) were performed in a 3-electrode setup, including as working electrode a platinum macroelectrode $(1.7 \mathrm{~mm}$ in diameter), polished before each experiment, a platinum wire was used as counter electrode and an AgCl-coated $\mathrm{Ag}$ wire as reference electrode. All solutions were degassed with nitrogen for $20 \mathrm{~min}$ prior to electrochemical characterization.

\section{Spectroelectrochemistry}

Spectroelectrochemical measurements were performed in a quartz cuvette ( $1 \mathrm{~mm}$ optical path) equipped with a platinum grid as working electrode, a platinum wire as counter electrode and an AgCl-coated Ag wire as reference electrode. The cell was filled with AR solution $\left(10^{-4} \mathrm{~mol} \mathrm{~L}^{-1}\right)$, kept in the dark, and degassed for $20 \mathrm{~min}$ by nitrogen bubbling prior to experiment. Spectroscopic data were obtained with a UV-visible spectrophotometer (Varian Cary $100 \mathrm{Scan}$ ) within the 400-650 nm window at $600 \mathrm{~nm} \mathrm{~min} \mathrm{mi}^{-1}$ and a spectrum was collected every minute. Buffer and AR solutions were measured concomitantly as reference and analysed sample, respectively. Oxidation and subsequent reduction of the AR solution were performed by applying first $+0.5 \mathrm{~V}$ for $10 \mathrm{~min}$. and then $-0.8 \mathrm{~V}(v s$. $\mathrm{Ag} / \mathrm{AgCl})$ for $15 \mathrm{~min}$. These durations were established according to the evolution and stability of the spectroscopic signals. Comparable experiments were also conducted using a fluorescence spectrophotometer (Varian Cary Eclipse) as reported previously. ${ }^{30}$ The excitation wavelength was $530 \mathrm{~nm}$ (slit width: $5 \mathrm{~nm}$ ) and the fluorescence emission spectra were collected between 550 and $750 \mathrm{~nm}$ (slit width: $5 \mathrm{~nm}$ ) at a wavelength scan rate of $600 \mathrm{~nm} \min ^{-1}$ (0.1 s integration time).

\section{Confocal microscopy and electrochemistry}

A homemade tank $(V=1.5 \mathrm{~mL})$ was built to directly couple electrochemistry and confocal microscopy at the surface of the 
Pt working electrode (see Fig. 1). The electrode surface was directly observed through a $0.17 \mathrm{~mm}$-thick coverslip for microscopy, constituting the bottom of the tank. Needles were inserted to flow nitrogen and degassed AR or RS solution $\left(2.5 \times 10^{-4} \mathrm{~mol} \mathrm{~L}^{-1}\right.$ each) within the tank. Images were collected with a laser scanning confocal microscope (Leica SP5), using a $5 \times$ magnification objective. RS fluorescence was excited at $514 \mathrm{~nm}$ with an argon laser and detected with a photomultiplier within the 549-688 nm window. To avoid excess exposition and photo-bleaching of the sample, images were taken under non-continuous exposition, typically an image every 8 s. The acquired images were displayed using a predefined colour look-up table (LUT) in order to highlight the fluorescence of RS.

\section{Results and discussion}

The cyclic voltammograms (CV) recorded at a platinum working electrode in a buffered aqueous solution (PBS pH 7.4) containing $2.5 \times 10^{-4} \mathrm{~mol} \mathrm{~L}^{-1}$ of either AR or RS are presented in Fig. 2a. These CVs were collected at a scan rate of $0.05 \mathrm{~V} \mathrm{~s}^{-1}$ and the potential was first swept in the negative direction from 0 to $-0.85 \mathrm{~V}$ vs. $\mathrm{Ag} / \mathrm{AgCl}$. Within this potential window, $\mathrm{RS}$ is reduced reversibly to $\mathrm{DH}$ and the $\mathrm{CV}$ exhibits a well-defined pair of peaks centred at $-0.50 \mathrm{~V}$ and $-0.25 \mathrm{~V} v$ s. $\mathrm{Ag} / \mathrm{AgCl}$ (blue dash line). The former peak is assigned to the $2 \mathrm{e}^{-}$-reduction whereas the latter corresponds to the DH re-oxidation to RS. The difference of potentials between both peaks is about $0.2 \mathrm{~V}$ when the $\mathrm{CV}$ is acquired at $0.05 \mathrm{~V} \mathrm{~s}^{-1}$ and appears to be very sensitive to the scan rate. This behaviour features a quasi-reversible electron transfer process. The dependence of the peak current on the sweep rate reveals a diffusion-limited mechanism as the intensity evolves linearly with the square root of the scan rate.

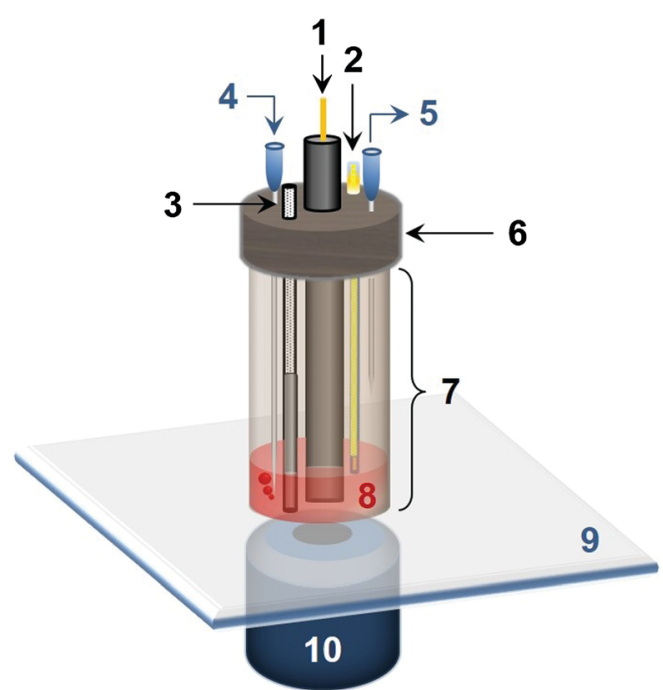

Fig. 1 Design of the homemade electrochemical cell for recording in situ fluorescence microscopy images under electrochemical control. Set of working, auxiliary and reference electrodes (1-3), needles for degassing $(4,5)$, cell cap (6), plastic cell core (7), solution (8), glass slide (9) and microscopy objective (10).
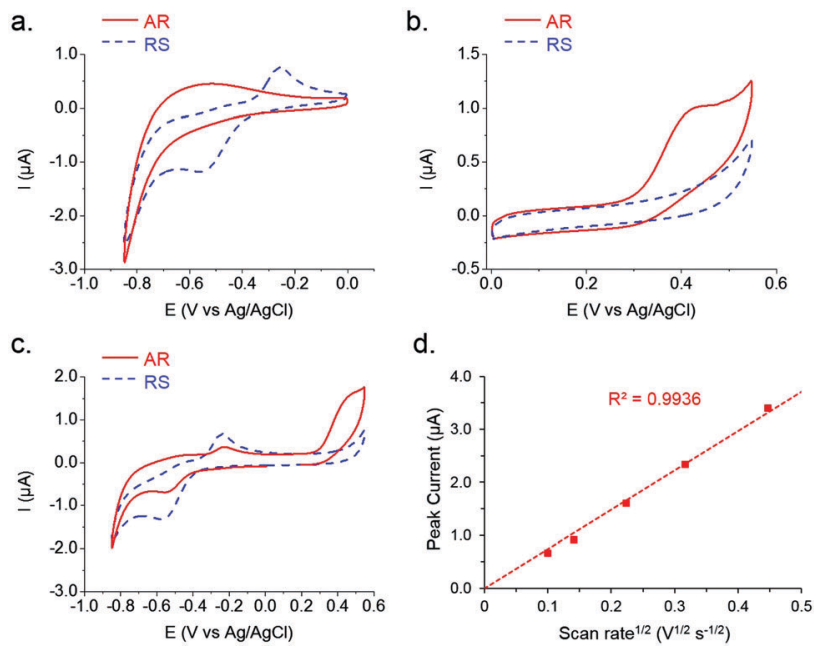

Fig. 2 Cyclic voltammograms (CVs) of $2.5 \times 10^{-4} \mathrm{~mol} \mathrm{~L}^{-1}$ AR (red solid line) and RS (blue dash line) recorded at a Pt disk electrode in buffered aqueous solution $\left(\mathrm{pH}\right.$ 7.4). Scan rate $0.05 \mathrm{~V} \mathrm{~s}^{-1}(\mathrm{a}-\mathrm{c})$. Linear relationship between the anodic peak current intensity and the square root of the scan rate (d).

By comparison, AR appears to undergo an irreversible reduction starting at $\sim-0.65 \mathrm{~V} v s$. $\mathrm{Ag} / \mathrm{AgCl}$ but the sweep back to $0 \mathrm{~V}$ only shows a large anodic shoulder at $\sim-0.50 \mathrm{~V} v$ s. Ag/AgCl (Fig. 2a, red solid line). When recording the $\mathrm{CV}$ of $\mathrm{AR}$ in such a potential window, the absence of well-identified faradaic signals indicates that RS is not formed under these electrochemical conditions. Such a result contrasts markedly with the work of GajovicEichelmann et al. wherein RS was formed electrochemically from $\mathrm{AR}$ in a similar experiment. ${ }^{31}$ However, this latter study was performed in McIlvaine buffer at $\mathrm{pH} 5$ instead of phosphate buffer at physiological $\mathrm{pH}$ in the present case, which is optimal for many enzymatic assays. It is noteworthy that $\mathrm{RS} / \mathrm{DH}$ redox couple is indeed very sensitive to $\mathrm{pH}$ as this system undergoes a two-electron transfer coupled with two protons. Regardless this proton transfer, it is also essential to take into account the acid/base equilibrium of RS. For that, a spectroscopic titration was conducted by recording the absorption spectra of RS solutions at various $\mathrm{pH}$ values in Britton-Robinson buffers. The corresponding $\mathrm{p} K_{\mathrm{a}}$ was found to be 5.95 which is compatible with previous data indicating a $\mathrm{p} K_{\mathrm{a}}$ of about $6.1 .^{27}$ Since our study is conducted above the $\mathrm{p} K_{\mathrm{a}}$ value, this may explain the observed discrepancy with the one of Gajovic-Eichelmann et al., especially because these molecules exhibit low solubility under acidic conditions. Also, the oxidized and reduced forms may have very distinct solubility according to their protonation state. It is also noteworthy that the $\mathrm{p} K_{\mathrm{a}}$ value varies markedly between $\mathrm{AR}$ and RS. According to a recent study, the first $\mathrm{p} K_{\mathrm{a}}$ of AR is 8.5 meaning that this molecule exists predominantly in its neutral form at the physiological $\mathrm{pH}$ used in the present work. ${ }^{32}$

Fig. $2 \mathrm{~b}$ gathers the CVs recorded for RS and AR between $0 \mathrm{~V}$ and $+0.55 \mathrm{~V} v s$. $\mathrm{Ag} / \mathrm{AgCl}$. In the case of RS, absolutely no peak is observed, unlike for AR which clearly features an irreversible anodic conversion with a peak centred at $+0.40 \mathrm{~V} v s$. Ag/AgCl.

This oxidation step appears to be essential for the formation of RS molecule from an AR solution as revealed by the CV 
obtained when scanning the potential in the full range, i.e. between $+0.6 \mathrm{~V}$ and $-0.9 \mathrm{~V}$ vs. $\mathrm{Ag} / \mathrm{AgCl}$ (Fig. 2c). In fact, when the potential is first swept toward the cathodic region and then in the anodic domain, AR is converted to $\mathrm{RS}$ as the typical faradaic features are observed. Under the experimental conditions tested, the electrochemical responses of AR and RS are diffusioncontrolled as inferred from the scan rate study (Fig. 2d). The diffusion coefficient of AR can be estimated from the slope of the plot reporting the oxidation peak current versus the square root of the scan rate. This calculation is based on the hypothesis of a 2-electron transfer step followed by a chemical reaction hypothesized to be a disproportionation. ${ }^{32}$ It leads to a value of $D_{\mathrm{AR}}=4.8 \times 10^{-6} \mathrm{~cm}^{2} \mathrm{~s}^{-1}$ which seems reasonable. It is noteworthy that this diffusion coefficient appears to be slightly higher than the values obtained for RS or RZ. ${ }^{30}$ To summarize this electrochemical investigation, it is concluded that the conversion of AR to RS proceeds through a direct oxidation step, and not via a reduction route. However, it is noticeable that electrochemical characterization only gives a partial information in the case of fluorogenic dyes because it solely reveals the faradaic exchange at the solution/electrode interface. To circumvent such a limitation, it is therefore necessary to record in situ spectroelectrochemical experiments in order to assign without ambiguity the nature of the electrogenerated species.

The spectroelectrochemical experiments were conducted in a $1 \mathrm{~mm}$ pathlength thin layer quartz cell filled with a $10^{-4} \mathrm{~mol} \mathrm{~L}^{-1}$ AR solution. A platinum gauze was used as a working electrode positioned in front of the optical window and absorption spectra were recorded under polarization. The result of this series of experiments is given in Fig. 3. First, the potential was directly stepped from the open circuit potential to $+0.50 \mathrm{~V} v s$. $\mathrm{Ag} / \mathrm{AgCl}$ and spectra were recorded every minute. The initial spectrum collected between $400 \mathrm{~nm}$ and $650 \mathrm{~nm}$ did not show any absorption in the visible region as expected from a perfectly colourless AR solution. The colour of the solution immediately
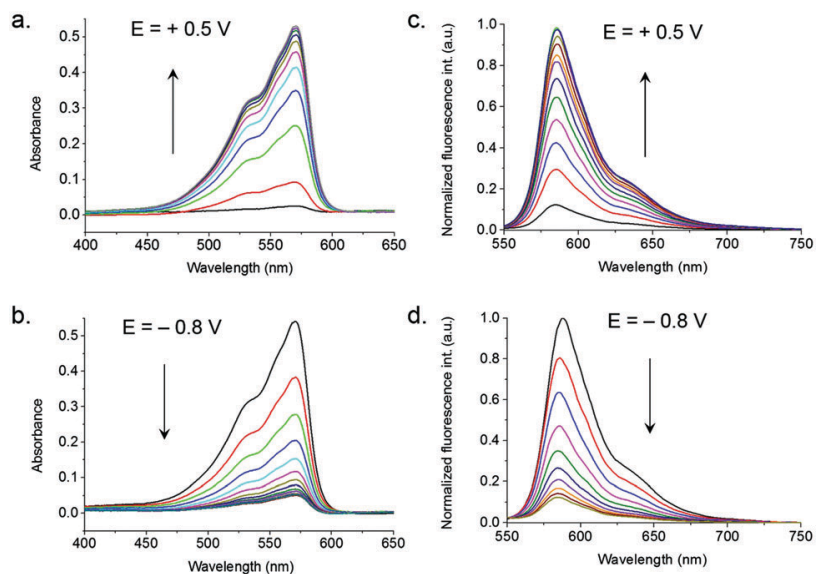

Fig. 3 Time evolution of the in situ absorption spectra recorded in a $10^{-4} \mathrm{~mol} \mathrm{~L}{ }^{-1}$ AR solution at a Pt mesh electrode. The electrode potential was sequentially held at $+0.5 \mathrm{~V} v \mathrm{vs}$. $\mathrm{Ag} / \mathrm{AgCl}$ for at least $10 \mathrm{~min}$ (a) and -0.8 $v s$. $\mathrm{Ag} / \mathrm{AgCl}$ for the same duration (b). Time evolution of the in situ fluorescence emission spectra recorded in the same conditions with an excitation wavelength of $530 \mathrm{~nm}$ (c and d, respectively). turned pink as soon as the anodic potential was applied and the corresponding spectra revealed a sharp absorption with a maximum centred at $\sim 570 \mathrm{~nm}$ with also a marked shoulder at $\sim 530 \mathrm{~nm}$ (Fig. 3a). These characteristic features can be safely assigned to RS as the typical shape and wavelength of the obtained spectra fit perfectly to literature data. ${ }^{1}$ The absorption intensity increased with time during the course of the chronoamperometry and then reached a plateau after about 10 minutes with an absorbance of $A_{\lambda=570 \mathrm{~nm}} \sim 0.6$. From the latter value, the amount of electrogenerated RS dye can be calculated and the concentration reached in situ is in good agreement with the initial AR concentration $\left(10^{-4} \mathrm{~mol} \mathrm{~L}^{-1}\right)$ and points toward a quantitative formation of RS. Interestingly, the applied potential can be directly stepped to a cathodic value such as $-0.80 \mathrm{~V} v s$. $\mathrm{Ag} / \mathrm{AgCl}$ from that solution. Then, the overall spectra intensity decreases steadily until the absorption due to RS almost completely vanishes because of the reversible reduction to the $\mathrm{DH}$ uncoloured form (Fig. 3b). The residual absorbance after 10 minutes was lower than 0.05 at the maximum wavelength, which corresponds to less than $10 \%$ of the initial value. This control experiment confirms that the conversion from RS to DH is indeed quantitative. The conclusion drawn from this spectroelectrochemical study is that the in situ electrochemical generation of RS from the AR solution is directly driven by a mild oxidation at $+0.50 \mathrm{~V} v s$. $\mathrm{Ag} / \mathrm{AgCl}$ and does not require any prior reduction step.

A very comparable series of spectroelectrochemical experiments were also conducted by recording the time evolution of fluorescence emission spectra. These spectra were collected with an excitation wavelength of $530 \mathrm{~nm}$ in order to directly monitor the formation of RS because AR is obviously non-fluorescent. The first potential step was performed by applying $+0.50 \mathrm{~V} v$ s. $\mathrm{Ag} / \mathrm{AgCl}$ (Fig. 3c). The corresponding spectra recorded every minute show a marked increase of the fluorescence intensity with the typical feature assigned to RS (maximum at $\lambda=590 \mathrm{~nm}$ ). The evolution of the signal slows down gradually and becomes then constant after about $11 \mathrm{~min}$. when the electrochemical conversion is completed inside the volume of the cell in front of the optical window. As the conversion is quantitative, the solution does not contain any more $\mathrm{AR}$, but RS instead at a concentration of $10^{-4} \mathrm{~mol} \mathrm{~L}^{-1}$. The second potential step was a reverse bias at $-0.80 \mathrm{~V}$ vs. Ag/AgCl (Fig. 3d). A spectrum was also recorded every minute and the time evolution evidenced a decrease of the fluorescence intensity as expected for the cathodic conversion of RS to DH. To summarize, the spectroelectrochemical experiments both in electronic absorption and fluorescence emission are perfectly self-consistent and confirm the hypothesis of a direct anodic generation of RS from AR established initially by the electrochemical investigation.

The direct generation of RS at the electrode surface in the conditions determined above was finally demonstrated by achieving the electrochemistry under confocal imaging of the electrode. The corresponding results are gathered in Fig. 4. For that, we designed a homemade electrochemical cell that can be ideally positioned in front of the objective of a confocal microscope. The design of the cell is briefly described in the experimental section and given in Fig. 1. It is basically a 
a.

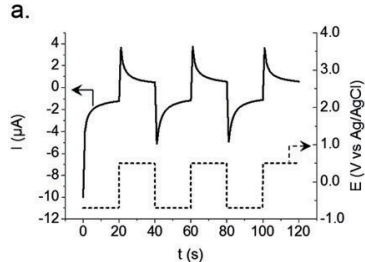

c.

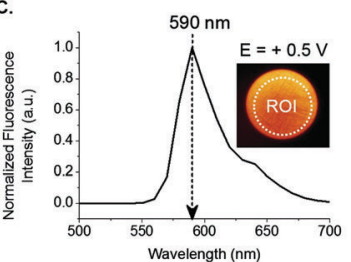

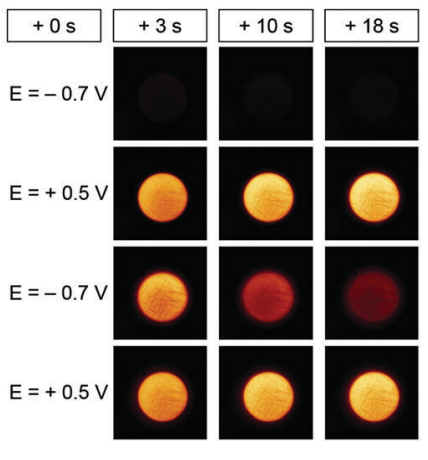

Fig. 4 Chronoamperogram (CA) recorded in a $2.5 \times 10^{-4} \mathrm{~mol} \mathrm{~L}^{-1} \mathrm{AR}$ solution at a Pt disk electrode (a). Time series of fluorescence images recorded in situ during the course of the CA (b). Each potential step lasts for $20 \mathrm{~s}$ and images are acquired $3 \mathrm{~s}, 10 \mathrm{~s}$ and $18 \mathrm{~s}$ after changing the potential value, respectively. Wavelength scan constructed from a series of fluorescence images of the electrode taken at different wavelength bandwidths (c). Inset showing the region of interest $(\mathrm{ROI})$ used to collect the relative fluorescence intensity.

straightforward way to collect microscopy data under electrochemical control as the setup comprises a set of electrodes and an optical window at the bottom part in order to record in situ microscopy imaging. A series of fluorescence images were collected during the course of a chronoamperometry. In this experiment, a $2.5 \times 10^{-4} \mathrm{~mol} \mathrm{~L}^{-1}$ solution of AR was filled in the cell and the potential applied at the working electrode was changed several times. Fluorescence images were collected upon excitation at $514 \mathrm{~nm}$, which is suitable for RS fluorophore. In this experiment, the applied potential was sequentially set alternatively from $-0.70 \mathrm{~V}$ to $+0.50 \mathrm{~V}$ vs. Ag/AgCl. Each potential step lasted for $20 \mathrm{~s}$ (Fig. 4a) and images of the fluorescence generation at the electrode surface were collected $3 \mathrm{~s}, 10 \mathrm{~s}$ and $18 \mathrm{~s}$ after changing the potential value (Fig. 4b). The first potential step at $-0.70 \mathrm{~V} v s$. $\mathrm{Ag} / \mathrm{AgCl}$ did not result in any recordable fluorescence, in contrast to the first step at $+0.50 \mathrm{~V}$ which was characterized by immediate appearance of a bright emission. The corresponding fluorescence intensity was stable and did not decrease over time until the next cathodic step. The latter showed a progressive diminution of the overall emission intensity after $10 \mathrm{~s}$ and $18 \mathrm{~s}$ since both the initial (AR) and electrogenerated species $(\mathrm{DH})$ are not fluorescent. As expected, the next positive step at $+0.50 \mathrm{~V} v s$. $\mathrm{Ag} / \mathrm{AgCl}$ re-established immediately a fluorescence level identical to that of the first anodic pulse (Fig. 4b). This series of results collected by in situ fluorescence microscopy under electrochemical control confirms directly the data observed during the initial electrochemical characterization underlying the necessity of an oxidation step prior to observing RS in solution from an AR solution.

Finally, the fluorescence microscope used is also equipped with a wavelength selector in excitation and emission which allows to perform a wavelength scan experiment. For that, a series of optical images were taken with a narrow emission bandwidth of $10 \mathrm{~nm}$ and centred at a given wavelength value. The fluorescence intensity extracted from a region of interest

(ROI) positioned on the electrode surface allows to reconstruct indirectly a full emission spectrum from the series of confocal images (Fig. 4c). Such a wavelength scan directly validates the nature of the chemical species generated as the spectrum is reminiscent of $\mathrm{RS}$ with a maximum at $\lambda \sim 590 \mathrm{~nm}$ at the studied concentration.

\section{Conclusions}

AR is a very useful fluorogenic tool that has been employed for more than two decades in many biochemical assays. This dihydroxyphenoxazine molecule is uncoloured and non-fluorescent but can be converted to RS which is a pink dye and fluorescent hydroxylphenoxazin-one. Typical assays consist in monitoring the fluorescence raise which reports either enzymatic processes or the presence of various chemical species such as ROS. However, the stability of this redox-sensitive indicator is influenced by interfering molecules. The electrochemical characterization of AR and RS solution at pH 7.4 are compared in order to establish exactly the conditions of formation of RS from AR. Moreover, various in situ techniques such as absorption and emission spectroelectrochemistry and also confocal fluorescence microscopy were recorded under electrochemical control. The cross-comparison between these approaches allows to study simultaneously the faradaic processes involving an electron transfer step and fluorescence signal that reveals the presence of the RS dye only under anodic polarization of the electrode surface. This result contrasts with previous works which reported the generation of RS following a reduction of AR. This study also proves that in situ microscopy is a technique of choice and a very powerful tool to infer molecular mechanism involving fluorogenic species, providing chemical and spatial information on electrochemical reactivity. Finally, another advantage of using confocal microscopy under electrochemical control relies on the establishment of a steady-state diffusion layer that allows a rapid recording of the fluorescence variation at the vicinity of the whole electrode surface. This allows to reveal the characteristic features of a fluorogenic dye more quickly than conventional spectroelectrochemistry which by contrast necessitates a bulk electrolysis.

\section{Acknowledgements}

The authors acknowledge the Centre National de la Recherche Scientifique (CNRS). This work was also funded by the French Agence Nationale de la Recherche (Project NO-SYNTHCELL under contract ANR-14-CE16-0015-02) and by CONACYT (project 208160).

\section{Notes and references}

1 C. Bueno, M. L. Villegas, S. G. Bertolotti, C. M. Previtali, M. G. Neumann and M. V. Encinas, Photochem. Photobiol., 2002, 76, 385.

2 G. V. Porcal, M. S. Altamirano, C. A. Glusko, S. G. Bertolotti and C. M. Previtali, Dyes Pigm., 2011, 88, 240.

3 R. S. Twigg, Nature, 1945, 155, 401. 
4 J. G. Mohanty, J. S. Jaffe, E. S. Schulman and D. G. Raible, J. Immunol. Methods, 1997, 202, 133.

5 M. Zhou, Z. Diwu, N. Panchuk-Voloshina and R. P. Haugland, Anal. Biochem., 1997, 253, 162.

6 M. Zhou and N. Panchuk-Voloshina, Anal. Biochem., 1997, 253, 169.

7 T. M. Kitson, Bioorg. Chem., 1998, 26, 63.

8 W. G. Gutheil, M. E. Stefanova and R. A. Nicholas, Anal. Biochem., 2000, 287, 196.

9 K. E. McElroy, P. J. Bouchard, M. R. Harpel, K. Y. Horiuchi, K. C. Rogers, D. J. Murphy, T. D. Y. Chung and R. A. Copeland, Anal. Biochem., 2000, 284, 382.

10 V. Mishin, J. P. Gray, D. E. Heck, D. L. Laskin and J. D. Laskin, Free Radical Biol. Med., 2010, 48, 1485.

11 S. G. Rhee, T.-S. Chang, W. Jeong and D. Kang, Mol. Cells, 2010, 29, 539.

12 B. Kalyanaraman, V. Darley-Usmar, K. J. A. Davies, P. A. Dennery, H. J. Forman, M. B. Grisham, G. E. Mann, K. Moore, L. J. Roberts, II and H. Ischiropoulos, Free Radical Biol. Med., 2012, 52, 1.

13 D. M. Amundson and M. Zhou, J. Biochem. Biophys. Methods, 1999, 38, 43.

14 M. J. Vazquez, B. Rodriguez, C. Zapatero and D. G. Tew, Anal. Biochem., 2003, 320, 292.

15 Q. Chen, E. J. Vazquez, S. Moghaddas, C. L. Hoppel and E. J. Lesnefsky, J. Biol. Chem., 2003, 278, 36027.

16 A. A. Starkov and G. Fiskum, J. Neurochem., 2003, 86, 1101.

17 F. L. Muller, Y. Liu and H. Van Remmen, J. Biol. Chem., 2004, 279, 49064.
18 B. Zhao, K. Ranguelova, J. Jiang and R. P. Mason, Free Radical Biol. Med., 2011, 51, 153.

19 B. Zhao, F. A. Summers and R. P. Mason, Free Radical Biol. Med., 2012, 53, 1080.

20 F. A. Summers, B. Zhao, D. Ganini and R. P. Mason, Methods Enzymol., 2013, 526, 1.

21 T. V. Votyakova and I. J. Reynolds, Arch. Biochem. Biophys., 2004, 431, 138.

22 K. J. Reszka, B. A. Wagner, C. P. Burns and B. E. Britigan, Anal. Biochem., 2005, 342, 327.

23 J. Serrano, M. Jove, J. Boada, M. J. Bellmunt, R. Pamplona and M. Portero-Otin, Biochem. Biophys. Res. Commun., 2009, 388, 443.

24 C.-W. Lee, Y.-C. Chen and A. Ostafin, J. Biomed. Nanotechnol., 2009, 5, 477.

25 J. B. Sambur, T.-Y. Chen, E. Choudhary, G. Chen, E. J. Nissen, E. M. Thomas, N. Zou and P. Chen, Nature, 2016, 530, 77.

26 S. Cakir and E. Y. Arslan, Chem. Pap., 2010, 64, 386.

27 S. Khazalpour and D. Nematollahi, RSC Adv., 2014, 4, 8431.

28 J. L. Lyon and K. J. Stevenson, Anal. Chem., 2006, 78, 8518.

29 H. H. Gorris and D. R. Walt, J. Am. Chem. Soc., 2009, 131, 6277.

30 T. Doneux, L. Bouffier, B. Goudeau and S. Arbault, Anal. Chem., 2016, 88, 6292.

31 N. Gajovic-Eichelmann and F. F. Bier, Electroanalysis, 2005, 17, 1043.

32 D. Debski, R. Smulik, J. Zielonka, B. Michalowski, M. Jakubowska, K. Debowska, J. Adamus, A. Marcinek, B. Kalyanaraman and A. Sikora, Free Radical Biol. Med., 2016, 95, 323. 